MAYO CLINIC Fy

\title{
A Videoconference Group Intervention to Improve Quality of Life of Cancer Caregivers:
}

\section{Lessons Learned}

\section{Maria I. Lapid MD, ${ }^{1}$ Melanie T. Gentry MD, ${ }^{1}$ Matthew M. Clark PhD,${ }^{1}$ Simon Kung MD, ${ }^{1}$}

Shehzad K. Niazi MD, ${ }^{2}$ Winston Tan MD, ${ }^{3}$ Pamela J. Atherton MS, ${ }^{4}$ Jeff A. Sloan PhD, ${ }^{4}$ Kevin J. Whitford MD, ${ }^{5}$ Joleen M. Hubbard MD, ${ }^{6}$ Andrea L. Cheville MD, ${ }^{7}$ Yolanda I. Garces MD, 8 Teresa A. Rummans MD ${ }^{1,2}$ Mayo Clinic Departments of ${ }^{1}$ Psychiatry \& Psychology, ${ }^{4}$ Health Sciences Research (Division of Biomedical Statistics \& Informatics), ${ }^{5}$ Internal Medicine, ${ }^{6}$ Medical Oncology, ${ }^{7}$ Physical Medicine \& Rehabilitation, ${ }^{8}$ Radiation Oncology, Mayo Clinic, Rochester, MN, USA Departments of ${ }^{2}$ Psychiatry \& Psychology, ${ }^{3}$ Hematology Oncology, Mayo Clinic, Jacksonville, FL, USA

\section{Background}

- Cancer caregiving can negatively impact the health and wellbeing of the caregiver.

- Interventions for reducing distress and improving coping skills have been shown to be effective in improving quality of life (QOL) for cancer caregivers.

- Telemedicine has the potential to help improve access for caregivers, given their patient care responsibilities.

- Study Aim: To determine feasibility and acceptability of a videoconferenced multidisciplinary intervention for cancer caregivers.
Methods

\section{- IRB-approved study}

- Setting: USA, Mayo Clinic

- Inclusion Criteria

- $\geq 18$ years of age

- Is a cancer caregiver defined as an unpaid individual who provides physical, practical, and/or emotional care and support to a cancer care recipient in the home or other healthcare setting.

- Has a care recipient $\geq 18$ years of age who has a malignancy diagnosis and undergoing or in the last 6 months has undergone cancer treatment.

\section{QOL Intervention}

- Structured multidisciplinary intervention of 8 videoconference group sessions provided over 4 weeks (Table 1).

- Groups were led by trained facilitators and included components of physical therapy, occupational therapy, psychosocial education, cognitivebehavioral intervention, supportive discussion, spiritual reflection, and mindfulness therapy.

- Feasibility was based on $25 \%$ recruitment, $80 \%$ session participation, and $80 \%$ questionnaire completion rates.

- QOL was assessed using LASA, a validated 0 to 10 item scale including overall QOL and individual QOL domains.

\section{Table 1. Multidisciplinary Intervention Session for Caregivers}

\begin{tabular}{|c|c|c|c|c|c|c|c|c|}
\hline & \multicolumn{8}{|c|}{ Session } \\
\hline Duration & 1 & 2 & 3 & 4 & 5 & 6 & 7 & 8 \\
\hline $2 \min$ & \multicolumn{8}{|c|}{ Check-in; AV check; Introduction; Review ground rules } \\
\hline $10 \mathrm{~min}$ & $\begin{array}{l}\text { Rapid Easy } \\
\text { Strength } \\
\text { Training } \\
\text { (REST) } \\
\text { [P] }\end{array}$ & $\begin{array}{l}\text { Relaxation \& } \\
\text { stress } \\
\text { management } \\
\text { training } \\
{[E, P, C]}\end{array}$ & $\begin{array}{l}\text { Rapid Easy } \\
\text { Strength Training } \\
\text { (REST) } \\
{[\mathrm{P}]}\end{array}$ & $\begin{array}{l}\text { Relaxation \& } \\
\text { stress } \\
\text { management } \\
\text { training } \\
{[\mathrm{E}, \mathrm{P}, \mathrm{C}]}\end{array}$ & $\begin{array}{l}\text { Rapid Easy } \\
\text { Strength } \\
\text { Training } \\
\text { (REST) } \\
{[\mathrm{P}]}\end{array}$ & $\begin{array}{l}\text { Relaxation \& } \\
\text { stress } \\
\text { management } \\
\text { training } \\
{[\mathrm{E}, \mathrm{P}, \mathrm{C}]}\end{array}$ & $\begin{array}{l}\text { Rapid Easy } \\
\text { Strength } \\
\text { Training } \\
\text { (REST) } \\
{[\mathrm{P}]}\end{array}$ & $\begin{array}{l}\text { Relaxation \& } \\
\text { stress } \\
\text { management } \\
\text { training } \\
{[\mathrm{E}, \mathrm{P}, \mathrm{C}]}\end{array}$ \\
\hline $28 \mathrm{~min}$ & $\begin{array}{l}\text { Defining } \\
\text { quality of } \\
\text { life } \\
{[E, P, C,} \\
\text { Sp, So, B] }\end{array}$ & $\begin{array}{l}\text { How to } \\
\text { recognize \& } \\
\text { avoid burnout } \\
\text { [E, P, C, Sp, } \\
\text { So, B] }\end{array}$ & $\begin{array}{l}\text { Symptom } \\
\text { management: } \\
\text { mood, sleep, } \\
\text { fatigue } \\
\text { [E, P, C, Sp, So, } \\
\text { B] }\end{array}$ & $\begin{array}{l}\begin{array}{l}\text { Emotional } \\
\text { coping } \\
\text { strategies for } \\
\text { caregivers } \\
{[\mathrm{E}, \mathrm{C}, \mathrm{Sp}, \mathrm{So}, \mathrm{B}]}\end{array} \\
\end{array}$ & $\begin{array}{l}\text { Spiritual } \\
\text { coping } \\
\text { strategies for } \\
\text { caregivers } \\
\text { [C, Sp, So, B] }\end{array}$ & $\begin{array}{l}\text { Communication } \\
\text { strategies with } \\
\text { patients \& } \\
\text { healthcare } \\
\text { providers } \\
{[\mathrm{E}, \mathrm{C}, \mathrm{So}]}\end{array}$ & $\begin{array}{l}\text { Advance } \\
\text { care planning } \\
{[E, P, C, S p} \\
\text { So, B] }\end{array}$ & $\begin{array}{l}\text { Psychosocial \& } \\
\text { community } \\
\text { caregiver } \\
\text { resources } \\
{[\mathrm{E}, \mathrm{So}, \mathrm{B}]}\end{array}$ \\
\hline $5 \mathrm{~min}$ & $\begin{array}{l}\text { Spiritual } \\
\text { Reflection } \\
\text { [Sp] } \\
\end{array}$ & $\begin{array}{l}\text { Mindfulness } \\
{[E, C]}\end{array}$ & \begin{tabular}{|l|} 
Spiritual \\
Reflection \\
[Sp] \\
\end{tabular} & $\begin{array}{l}\text { Mindfulness } \\
{[E, C]}\end{array}$ & $\begin{array}{l}\text { Spiritual } \\
\text { Reflection } \\
\text { [Sp] }\end{array}$ & $\begin{array}{l}\text { Mindfulness } \\
{[E, C]}\end{array}$ & \begin{tabular}{|l} 
Spiritual \\
Reflection \\
[Sp] \\
\end{tabular} & $\begin{array}{l}\text { Mindfulness } \\
{[E, C]}\end{array}$ \\
\hline
\end{tabular}

\section{Results}

\section{- Demographics}

- 20 caregivers enrolled

- $80 \% \geq 65$ years

- $76.5 \%$ female

- $88.2 \%$ spousal caregiver

- $100 \%$ Caucasian

- Highly educated

- Feasibility

- $60 \%$ attended $1-5$ sessions

- 15\% attended 6-8 sessions

- $25 \%$ attended no sessions

- QOL measures improved post-intervention (Figure 1)

\section{Figure 1. QOL Outcomes}

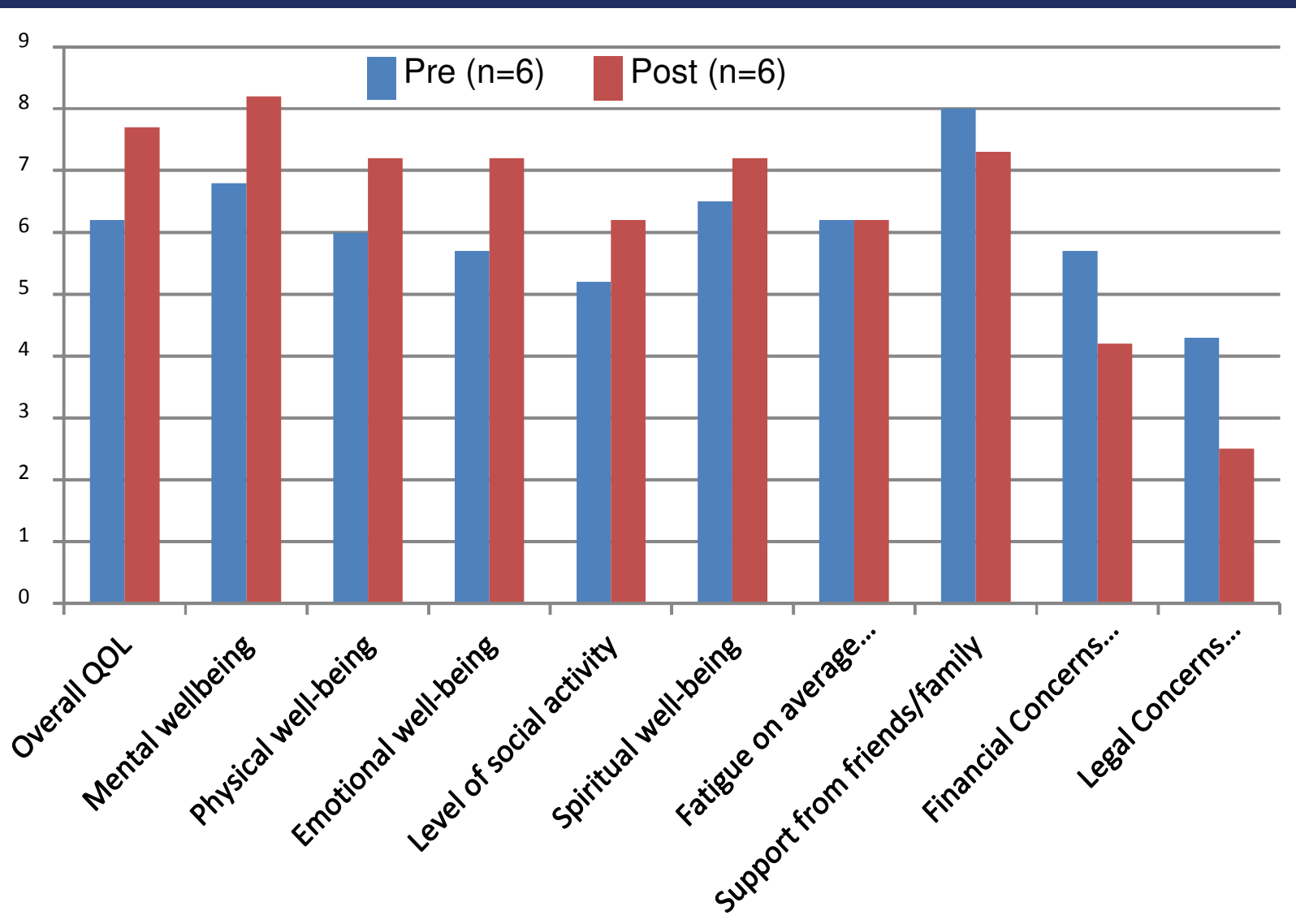

Conclusions

- Caregivers reported higher QOL in multiple domains after participating in intervention.

- Offering QOL interventions to caregivers through telehealth remains promising; however, more effective strategies are needed to overcome barriers to participation.

\section{References}

. Clark MM et al. Cancer 2013;119:880-7 2. DuBenske LL et al. Med Decis Making 2010;30:732-44

Northouse LL et al. CA Cancer J Clin 2010; 60:317-39

4. Rummans TA et al. J Clin Oncol 2006;24:635-42 5. Solberg Nes L et al. Lung Cancer 2012;77:611-6 6. Zulman DM et al. Psychooncology 2012;21:336-41 